\title{
hnRNP A1 interacts with the genomic and subgenomic RNA promoters of Sindbis virus and is required for the synthesis of $G$ and SG RNA
}

\author{
Hongxing Gui', Chi-Wei Lu², Sandra Adams ${ }^{3}$, Victor Stollarr ${ }^{4,5}$, Mei-Ling Li ${ }^{4 *}$
}

\begin{abstract}
Background: Sindbis virus (SV) is the prototype of alphaviruses which are a group of widely distributed human and animal pathogens. Heterogeneous nuclear ribonucleoprotein (hnRNP) A1 is an RNA-binding protein that shuttles between the nucleus and the cytoplasm. Our recent studies found that hnRNP A1 relocates from nucleus to cytoplasm in Sindbis virus (SV)-infected cells. hnRNP A1 binds to the 5' UTR of SV RNA and facilitates the viral RNA replication and translation.
\end{abstract}

Methods: Making use of standard molecular techniques, virology methods and an in vitro system developed by our lab to assess the role of hnRNP A1 in SV positive strand RNA synthesis.

Results: hnRNP A1 interacted with the genomic (G) and subgenomic (SG) RNA promoters. Knockdown of hnRNP A1 resulted in markedly decrease in the synthesis of $G$ and SG RNA both in infected cells and in vitro.

Conclusions: Our study provides the first direct evidence that hnRNP A1 actively participates in viral RNA replication and is required for the synthesis of G and SG RNA.

\section{Background}

Alphaviruses are a group of widely distributed human and animal pathogens that includes almost 40 currently known members [1]. In natural conditions, most alphaviruses are transmitted by mosquitos that develop lifelong chronic infections [2]. The presence of virus in the mosquito salivary glands mediates infection of vertebrates that serve as amplifying hosts. In vertebrates, alphaviruses develop acute infections characterized by high-titer viremia that is required for infection of mosquitoes during their blood meal. Ultimately, alphavirus infection of the vertebrate host is either cleared by the immune system or causes the death of an infected host.

Sindbis virus (SV) is the prototype virus of the family Togaviridae, genus alphavirus $[1,3,4]$. Sindbis virus has a non-segmented single-strand RNA genome of positive polarity, i.e. the genome can function as a messenger RNA. The genome is 11,703 nts in length, has a $5^{\prime}$ type 0 cap and is polyadenylated at its $3^{\prime}$ end.

\footnotetext{
* Correspondence: lime@umdnj.edu

${ }^{4}$ Department of Molecular Genetics, Microbiology \& Immunology, UMDNJRobert Wood Johnson Medical School, Piscataway NJ 08854, USA
}

In the infected cells, SV express only four nonstructural proteins, namely nsP1, nsP2, nsP3 and nsP4, which form, together with host factors, the enzyme complex responsible for the synthesis of virus-specific RNAs [5]. The nsP1 protein was found to have methyltransferase and guanylyltransferase activity, which are required for capping the newly synthesized, positive-strand viral RNAs [6-8]. It is palmitoylated and appears to be membrane-bound [9]. It also plays a role in the regulation of viral (-) strand RNA synthesis [10], and in the membrane attachment of the replication complex [11]. nsP2 was shown to be an RNA triphosphatase [12], RNA helicase [13] and a papain-like protease [14], and is responsible for the processing the P123 and P1234 polyproteins into the individual nsPs [3]. In addition, nsP2 is present not only in the replication complex, but was also found distributed in the cytoplasm and nuclei of infected cells $[15,16]$. nsP3 is the only nsP which is phosphorylated but its function is not yet clearly defined. The mutations in this protein can affect RNA replication, and, based on the structure of the replication complexes, this protein appears to have a structural function in the replication complex [17]. nsP4 is an 
RNA-dependent RNA polymerase (RDRP) [18] and possesses terminal adenylyltransferase activity [19].

Upon entry into the cell, the genome is translated into one of two polyproteins, P123 or P1234. These polyproteins are later processed in several steps by a viral protease into the four nonstructural (ns) proteins, nsP1, nsP2, nsP3, and nsP4. Initially, a complex of P123 (uncleaved nsP1, nsP2, and nsp3), and nsP4 form an RNA synthesizing complex that uses the incoming viral RNA as a template to synthesize a (-) strand RNA equivalent in length to the viral genome $[5,20]$. Upon further processing of the ns polyproteins, an RNAsynthesizing complex, now consisting of nsP1, nsP2, nsP3, and nsP4 is formed which uses the (-) strand RNA as a template to synthesize two (+) strand RNAs, a genomic (G) RNA, and a subgenomic (SG) RNA which has the same sequence as the terminal $3^{\prime}$ one third of the viral genome. The SG RNA serves as the message for the three structural proteins, the capsid or $C$ protein, and the two envelope proteins, E1 and E2. Because of stop codons after the nsP4 coding region, only the four ns proteins are translated from the G RNA.

In addition to virus-encoded factors, many steps in virus infections involve host factors. Such virus-host interactions are crucial determinants of virus host range, replication, and pathology, offer insights into viral and cellular function, and provide antiviral targets. Thus identifying such interactions and the associated host factors is a major frontier in virology [21,22]. Recently, using a human-genome-wide RNAi screen Krishnan et al. [23] have identified 305 host proteins that affect West Nile virus infection. Functional clustering of these genes revealed a complex dependence of this virus on a wide variety of host molecules and cellular pathways in viral infection. While the viral components of the SV replicase/transcriptase are known and to some extent characterized, the cellular proteins involved in viral RNA synthesis remain poorly understood. Moreover, while there is also strong evidence that host factors play a significant role in the activity of the replicase/transcriptase $[24,25]$, the cellular proteins associated with the alphavirus nsPs and RNA remain to be characterized. The first reported interaction between a host protein and viral RNA was that between the mosquito La autoantigen and the $3^{\prime}$ end of the SV negative strand RNA [26]. Recent work by Burnham et al. [27] has demonstrated that the host protein hnRNP $\mathrm{K}$ interacts with nsP2 and SG RNA in SV-infected cells. Several reports $[17,28,29]$ have identified other cellular proteins associated with the SV replicase/transcriptase. These include cytoskeleton proteins, ribosomal subunits, chaperones, and hnRNPs. However, the roles of these cellular proteins in SV replication remain unclear. hnRNP A1 was not identified in these studies.
Members of the hnRNP family (including hnRNP A1) are involved in IRES-dependent translation of cellular and viral mRNA [30-32]. Moreover, hnRNPs are involved in several RNA metabolic processes, such as pre-mRNA splicing and trafficking $[33,34]$ and telomerase regulation [35]. There is strong evidence that hnRNP A1 plays a critical role in telomere biogenesis [36]. Interestingly, hnRNP A1 has been shown to be involved in the replication of many viruses including mouse hepatitis virus [MHV], hepatitis $\mathrm{C}$ virus $[\mathrm{HCV}]$, dengue virus and human papillomavirus 16 [HPV 16], human cytomegalovirus, and vesicular stomatitis virus [VSV] [37-43].

Our recent studies reported that hnRNP A1 plays an essential role in the replication of SV [44]. hnRNP A1 interacted with the SV 5'UTR and facilitated the translation of viral RNA. Knockdown of hnRNP A1 reduced SV RNA synthesis and the yield of progeny virus. In the present study we extended the experiments on the role of hnRNP A1 in SV positive strand RNA synthesis. Our results suggest that hnRNP A1 associated with the genomic (G) and subgenomic (SG) RNA promoters and enhances the synthesis of $G$ and SG RNA.

\section{Methods}

\section{Cells and virus}

BSC40 cells were cultured at $37^{\circ} \mathrm{C}$ in Eagle's minimum essential medium supplemented with $10 \%$ fetal calf serum (FCS) (Mediatech). CEF (chick embryo fibroblast) cells were grown in Dulbecco's modified Eagle medium (DMEM) supplemented with 10\% FCS. SV was propagated in CEF cells. Cells were infected with SV at specified multiplicity of infection (moi) for $1 \mathrm{~h}$ at $34^{\circ} \mathrm{C}$, washed away unbound inoculum, and refed with fresh medium. Media from infected cultures were harvested at the indicated times, and titers of SV were measured by plaque formation on CEF cells. Recombinant vaccinia viruses encoding Sindbis virus P123, nsP4, or T7 polymerase (VTF7-3), were kindly provided by Drs. Charles M. Rice and Richard Hardy and were propagated in BSC40 cells as described previously [45]. The Sindbis virus P123 contained the N614D change in nsP2 that results in more rapid processing of P123 than is observed with the wt P123[20,46]. The various nsPs were expressed from a T7 promoter.

\section{Preparation of labeled RNA probes and binding assay}

24-mer and 45-mer oligoribonucleotides representing the negative strand sequence corresponding to nt 7579 to 7602 and nt 1-45 of the SV genome were synthesized by Dharmacon Research Inc. As already noted, these are the minimal sequences that have SG and G promoter activity respectively $[47,48]$. Oligoribonucleotides were labeled at their $5^{\prime}$ ends using polynucleotide kinase and $\left[\gamma_{-}{ }^{32} \mathrm{P}\right]$ ATP. 
An EMSA was carried out to determine the interaction between the promoters and hnRNP A1 as described previously [47]. Briefly, $2 \mu \mathrm{g}$ of hnRNP A1 was incubated for $30 \mathrm{~min}$ at $25^{\circ} \mathrm{C}$ with one of the ${ }^{32} \mathrm{P}$-labeled RNA probes $\left(1 \times 10^{4}\right.$ c.p.m). The reaction was carried out in binding buffer (10 mM Hepes, $\mathrm{pH} 7.5 / 150 \mathrm{mM} \mathrm{KCl} /$ $0.5 \mathrm{mM}$ EGTA/2 mM $\mathrm{MgCl}_{2} / 1 \mathrm{mM} \mathrm{DTT} / 1$ unit RNa$\sin / 10 \%$ glycerol) and the final volume of the reaction mixture was $10 \mu \mathrm{l}$. Binding of hnRNP A1 to the G and SG promoters was recognized by a slower migration of the labeled RNA probes.

\section{Northern blot analysis of G and SG RNA}

BSC40 cells were infected with SV at an moi of $40 \mathrm{pfu} /$ cell and at $8 \mathrm{~h}$ post infection total RNA was extracted using RNeasy mini kit (Qiagen). Five $\mu \mathrm{g}$ of total RNA was denatured with formaldehyde, and electrophoresed through a $1.5 \%$ agarose gel. The RNAs were transferred to a nylon membrane and subjected to northern blotting. The negative-strand probe, SV7772(-), which contains the negative-strand sequence from nt 7772 to 7754 were end-labeled with ${ }^{32}$ P- $\gamma$-ATP. After hybridization, the nylon membrane was washed twice with $2 \times$ SSC and $0.1 \%$ SDS, exposed to a storage phosphor screen and scanned on a Molecular Dynamics Typhoon Phosphorimager.

\section{Expression and preparation of recombinant Sindbis virus nonstructural proteins}

BSC40 monolayers in T75 tissue culture flasks were infected with three different recombinant vaccinia virus vectors; these encode the SV nonstructural polyprotein P123 (the polyprotein precursor of nsP1, nsP2, and nsP3), SV nsP4, and T7 RNA polymerase, each at an moi of one pfu/cell. Twenty to twenty four hours after infection, the P15 fraction was prepared according to Lemm et al. [45]. The P15 pellet isolated from one T75 flask was resuspended in $50 \mu \mathrm{l}$ of storage buffer $(10 \mathrm{mM}$ Tris-Cl [pH 7.8], $10 \mathrm{mM} \mathrm{NaCl}, 15 \%$ glycerol) and used as the source of the Sindbis virus replicase/transcriptase (R/T) complex.

\section{In vitro synthesis of SV RNA}

The reaction mixture for the in vitro synthesis of SV RNA $(25 \mu \mathrm{l})$ contained $5 \mu \mathrm{l}$ of $5 \times$ reaction buffer $(200 \mathrm{mM}$ Tris- $\mathrm{HCl}, \mathrm{pH} 7.9,30 \mathrm{mM} \mathrm{MgCl}{ }_{2}$, and $50 \mathrm{mM} \mathrm{NaCl}$ ), $10 \mathrm{mM}$ dithithreitol, 40 units of RNase inhibitor (Promega), $2 \mu \mathrm{g}$ negative-strand RNA which serves as promoter/template $(\mathrm{P} / \mathrm{T})$, and $12.2 \mu \mathrm{l}(5.4 \mu \mathrm{g}$ of protein $/ \mu \mathrm{l})$ of P15 extract prepared from BSC40 cells infected with recombinant vaccinia viruses expressing the T7 RNA polymerase, the SV polyprotein, P123, and the SV nsP4. Standard reaction mixtures contained $3 \mathrm{mM}$ ATP, $2 \mathrm{mM}$ UTP, $2 \mathrm{mM}$ CTP, and $0.5 \mathrm{mM}$ GTP. $\left[{ }^{32} \mathrm{P}\right] \alpha-\mathrm{GTP}(800 \mathrm{Ci} / \mathrm{mM}$,
$10 \mu \mathrm{Ci} / \mu \mathrm{l})$ was included to label the transcripts. Incubation was at $37^{\circ} \mathrm{C}$ for 1 hour. RNA was extracted using phenolchloroform and electrophoresed on a $1 \%$ denaturing agarose gel containing 2.2 $\mathrm{M}$ formaldehyde. The gel was transferred to a Genescreen Plus Hybridization Transfer Membrane (Perkin Elmer); the membrane was then exposed to a storage phosphor screen and scanned as described previously [49]. The negativestrand $\mathrm{P} / \mathrm{T}$ for making both $\mathrm{G}$ and SG RNA was as described in Li et al. [48].

\section{Expression and purification of recombinant hnRNP A1 protein}

hnRNP A1 cDNA derived from SF268 cellular mRNA was cloned into pET30a and expressed as described [44]. The recombinant hnRNP A1 protein was purified using a HisTrap kit (GE). Protein purity was determined by electrophoresis on $12 \%$ SDS-PAGE, and concentration was determined using the Bio-Rad protein assay.

\section{siRNA knockdown}

BSC40 cells were maintained in antibiotic-free media. $100 \mathrm{nmol}$ of siRNA targeting hnRNP A1 (ON-TARGETplus SMARTpool L-008221-00-0005, Dharmacon) was transfected along with $3 \mu \mathrm{l}$ FuGENE HD transfection reagent (Roche) in $0.4 \mathrm{ml}$ MEM supplemented with $10 \%$ FCS following manufacturer's directions. Three days after transfection cells were co-infected with recombinant vaccinia viruses expressing T7, SV-nsP123 and SV-nsP4 at an moi of one pfu/cell. P15 fraction was prepared as described [47]. An aliquot of cell lysate was subject to Western blot for analysis of protein expression.

\section{Western blotting}

Expression of hnRNP A1 was examined by Western blotting using anti-hnRNP A1 antibody (Abcam). Briefly, cells were lysed in sample buffer and proteins were fractionated by SDS-PAGE in $12 \%$ polyacrylamide gels and transferred to PVDF membranes by wet transfer. Membranes were blocked with PBS containing 5\% low-fat dry milk. Anti-hnRNP A1 mouse antibody was then added, and the membranes were washed with PBS containing $0.2 \%$ Tween 20 . Goat anti-mouse horseradish peroxidase-conjugated antibody (BioRad) and the ECL kit (Pierce) were used to detect bound antibodies.

\section{Results}

\section{hnRNP A1 interacts with the G and SG promoters of} Sindbis virus

Our previous study demonstrated that knockdown of hnRNP A1 in infected cells reduces the viral RNA synthesis [44]. To further dissect the role of hnRNP A1 in viral RNA synthesis, we tested if hnRNP A1 is 


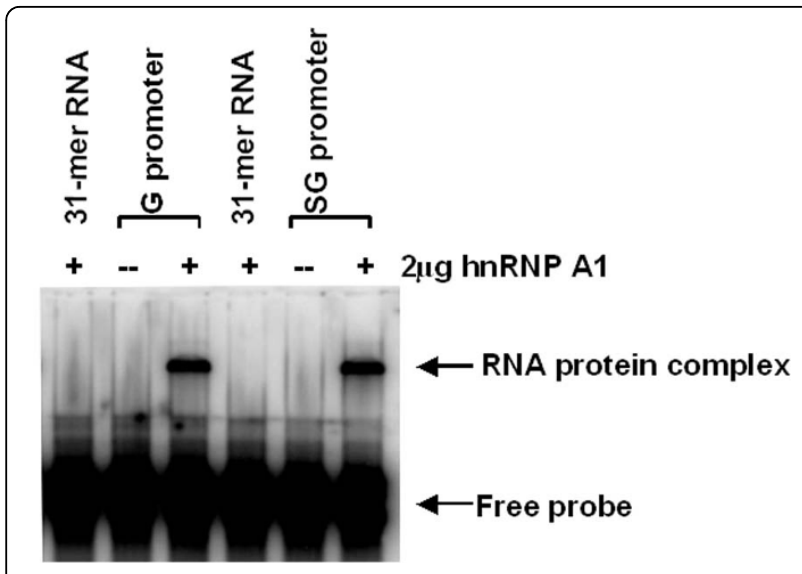

Figure 1 Binding of hnRNP A1 to the G and SG promoters. EMSA was performed as described under Material and Methods. The $\mathrm{G}$ and SG promoters were end-labeled with ${ }^{32} \mathrm{PO}_{4}\left(1 \times 10^{4}\right.$ $\mathrm{cpm}$ per reaction) and incubated at $37^{\circ} \mathrm{C}$ for 30 min either alone or with $2 \mu \mathrm{g}$ of hnRNP A1. A non-specific 31-mer RNA oligo was endlabeled with ${ }^{32} \mathrm{PO}_{4}$ and reacted with $2 \mu \mathrm{g}$ of hnRNP A1

involved in the positive strand RNA synthesis by interacting with the promoters. To learn whether hnRNP A1 interacts with the $\mathrm{G}$ and $\mathrm{SG}$ promoters, we tested the binding of hnRNP A 1 to the SV G and SG promoters. An EMSA was carried out using hnRNP A1 and the labeled G and SG promoters of Sindbis virus. As shown in Fig. 1, hnRNP A1 did not bind to a non-specific 31mer RNA oligo but did bind to the SV G and SG promoters slowing their electrophoretic mobility. These results indicate that the interaction between the $G$ and SG promoters and hnRNP A1 is specific.
hnRNP A1 is required for the synthesis of G and SG RNA in infected cells

While we have good evidence that hnRNP A1 binds to the $\mathrm{G}$ and $\mathrm{SG}$ promoters, the consequences of this interaction for viral RNA synthesis are not known. Our recent studies showed that hnRNP A1 re-localizes from nucleus to cytoplasm of SV-infected cells. Knockdown of hnRNP A1 inhibits the virus replication [44]. These findings led us to hypothesize that the binding of hnRNP A1 to the G and SG promoters is involved in the regulation of G and SG RNA synthesis during the virus replication cycle. To assess the role of hnRNP A1 in viral positive strand RNA synthesis, we knocked down the expression of hnRNP A1 in infected cells and examined the synthesis of G and SG RNA by Northern blot. hnRNP A1 was depleted by siRNA targeting hnRNP A1 in BSC40 cells as described previously [44]. Knockdown efficiency was examined by Western blot using anti-hnRNP A1 antibody (Abcam). As shown in Fig. 2A, expression of hnRNP A1 was diminished in cells transfected with siRNA targeting hnRNP A1. Cells were then infected with SV at an moi of $40 \mathrm{pfu} /$ cell for $8 \mathrm{~h}$. Total RNA was extracted and subject to Northern blot as described in Materials \& Methods. As shown in Fig. 2B, the synthesis of G and SG RNA was dramatically inhibited when hnRNP A1 was depleted indicating hnRNP A1 is essential for the positive strand RNA synthesis. The medium was collected and plaque assay was carried out to determine the virus titer, as shown in Fig. 2C, the titer dropped about $4 \log$ in hnRNP A1 knockdown cells.
A.

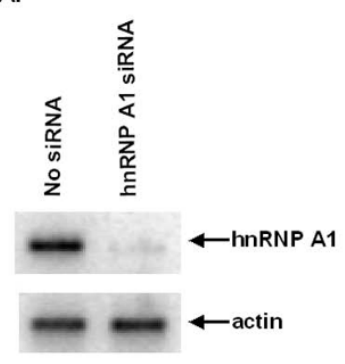

B.

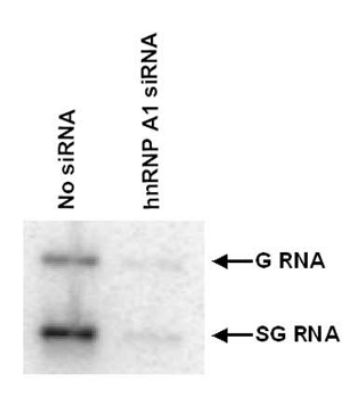

C.

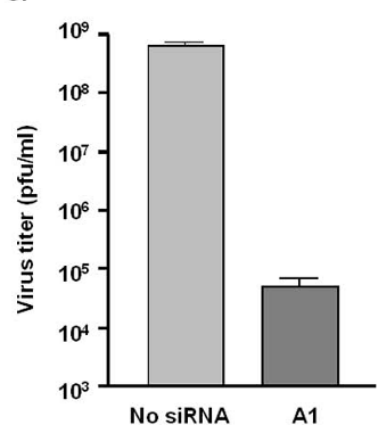

Figure 2 Effect of hnRNP A1 on the synthesis of G and SG RNA in infected cells. (A) Western blot to examine the expression of hnRNP A1. hnRNP A1 was depleted by siRNA knockdown technique as described under Materials and Methods. Cell lysates were prepared 3 days after transfection of siRNA. Western blot was carried out using antibody to hnRNP A1. Expression of actin was examined by Western blot as a control. (B) Northern blot to detect G and SG RNA in infected cells. Cells were transfected with no siRNA or siRNA targeting hnRNP A1 for 3 days. Cells were infected with SV at an moi of $40 \mathrm{pfu} / \mathrm{cell}$ for $8 \mathrm{~h}$. Total RNA were extracted and electrophoresed through an agarose gel, transferred to a nylon membrane, and characterized by Northern blot analysis, using ${ }^{32}$ P-labeled negative-strand probe SV7772(-). (C) Replication of SV in hnRNP A1-depleted cells. Cells treated or untreated with siRNA targeting hnRNP A1 were infected with SV at an moi. of 40 pfu/cell and incubated at $34^{\circ} \mathrm{C}$. Medium was harvested $8 \mathrm{~h}$ p.i. and assayed for infectious virus by plaque formation on CEF cells. Mean values and standard errors from duplicate samples are shown. 


\section{hnRNP A1 enhances the synthesis of G and SG}

\section{RNA in vitro}

We made use of an in vitro system for the synthesis of $G$ and SG RNA developed by our laboratory $[48,49]$ to examine the role of hnRNP A1 in the synthesis of G and SG RNA. Synthesis of the G and SG RNA transcript was followed by including ${ }^{32} \mathrm{P}-\alpha$-labeled GTP in the reaction mixture, along with the four unlabeled NTPs, the P/T, and the P15 fraction as described in Materials \& Methods.

The level of endogenous hnRNP A1 in BSC40 cells was knocked down by siRNA targeting of hnRNP A1 as described in Materials \& Methods. The knockdown efficiency was confirmed by Western blot using anti-hnRNP A1 antibody (data not shown). The hnRNP A1-depleted BSC40 cells were then co-infected with recombinant vaccinia viruses expressing $\mathrm{T} 7$ polymerase, SV P123 and SV nsP4 and the p15 were prepared as described [47]. As shown in Fig. 3A the expression of nsP4 in hnRNP A1-depleted cells was as good as in wild type cells indicating that knockdown of hnRNP A1 did not affect the expression of the SV nonstructural proteins. To see if knockdown of hnRNP A1 affects G and SG RNA synthesis in vitro, the reaction was set up as described [48] using either normal or hnRNP A1depleted replicase/transcriptase (P15 fraction) and the $\mathrm{P} / \mathrm{T}$ which contains both $5^{\prime}$ and $3^{\prime}$ termini and the SG promoter region. As shown in Fig. $3 \mathrm{~B}$ depletion of hnRNP A1 in BSC40 cells resulted in decreased synthesis of both G and SG RNA. Addition of purified hnRNP A1 back to the hnRNP A1-depleted replicase/ transcriptase was able to restore the RNA synthesis. The results indicate that hnRNP A1 is required for $G$ and SG RNA synthesis.

\section{Discussion}

Host factors together with viral proteins regulate virus RNA replication. The finding that mutations in the viral genome sometimes have different effects in vertebrate cells and mosquito cells has suggested that cellular factors play a role in viral RNA synthesis [7]. Recent studies from several groups have identified cellular proteins associated with the SV RNA replication complex by isolating nonstructural protein-containing complexes from infected cells [17,27-29]. However, the roles that most of these proteins play in the replication of SV remain to be elucidated. Our recent study found that after SV infection hnRNP A1 relocalized from nucleus to cytoplasm, where the SV replication occurs. hnRNP A1 was able to bind to the capped 5'UTR of SV RNA and knockdown of hnRNP A1 diminished the SV viral RNA and protein synthesis, cap-dependent translation and viral replication. We therefore conclude that hnRNP A1 promotes SV replication via binding to the 5'UTR and facilitates possibly both viral RNA synthesis and translation [44].

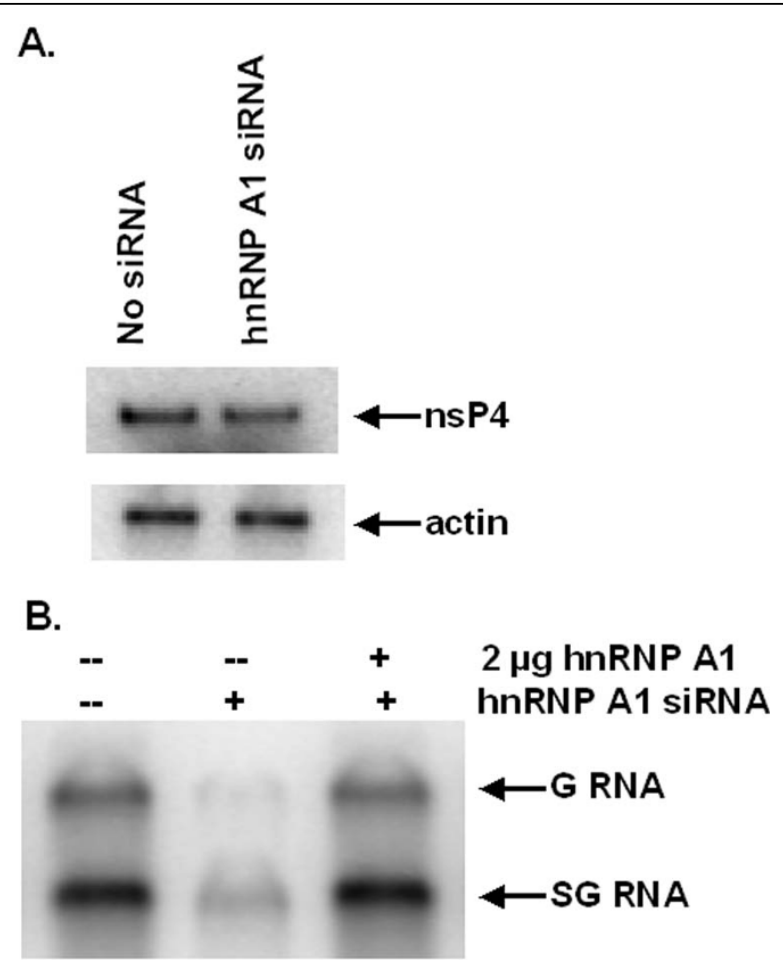

Figure 3 Effect of hnRNP A1 on the synthesis of G and SG RNA in vitro. (A) Western blot to examine the expression of nsP4. BSC40 cells were transfected with no siRNA or siRNA targeting hnRNP A1 for 3 days as described under Materials and Methods. Cells were coinfected with recombinant vaccinia viruses expressing T7 RNA polymerase, SV nsP123 and SV nsP4 at an moi of one for $24 \mathrm{~h}$. Cell lysates were prepared and subjected to 12\% SDS-PAGE electrophoresis. Western blot was carried out using antibody to nsP4. Expression of actin was examined by Western blot as a control. (B) in vitro G and SG RNA synthesis. hnRNP A1 was depleted from BSC40 cells as described above. Cells were coinfected with recombinant vaccinia viruses expressing T7 RNA polymerase, SV nsP123 and SV nsP4 and P15 fractions were prepared as described under Materials and Methods. $\mathrm{P} / \mathrm{T}$ were prepared and standard reaction conditions were used as described under Materials and Methods.

How does hnRNP A1 contribute to the replication of SV? As hnRNP A1 is an RNA-binding protein, we speculated that it might facilitate viral RNA synthesis via association with viral RNA. In our previous study we found that hnRNP A1 interacts with the 5'UTR of SV RNA and promotes the synthesis of negative strand RNA [[44], data not shown]. In the present study we demonstrated that hnRNP A1 specifically interacts with both the $G$ and SG promoters and enhances the synthesis of G and SG RNA as knockdown of hnRNP A1 rendered dramatically decrease in the synthesis of these two RNA species. Taken together we demonstrate that hnRNP A1 is required for the synthesis of both positive and negative strand RNA. 
G and SG promoters are different in sequences. Our previous study showed that nsP4, the RDRP, has distinct sites for the binding of G and SG promoters $[47,48]$. We therefore suggest that in a similar fashion, hnRNP A1 also has different sites for the recognition of these two promoters. In future work we intend to use the same procedures that were used to identify the $G$ and SG promoter binding sites in nsP4 [47] to identify the binding sites for $\mathrm{G}$ and SG promoters in hnRNP A1.

How does hnRNP A1 contribute to the viral RNA replication? hnRNP A1 is predominantly a nuclear protein. During SV infection nearly all of hnRNP A1 relocalizes to cytoplasm in association with viral RNA. Precisely what interactions lead to the recruitment of hnRNP A1 to the viral RNA replication complex is unknown. As hnRNP A1 is an RNA-binding protein, it may direct the viral RNA replication complex containing the viral RDRP, other viral and cellular factors to the sites where replication of viral RNA is initiated. Moreover, considering the reported physiological roles of hnRNP A1, we speculate that hnRNP A1 may interact with viral nonstructural proteins which comprise the viral RNA replication complex and facilitates the viral RNA replication. Further investigation is going on to address how hnRNP A1 contributes to viral RNA replication.

To our knowledge, this is the first report to describe an RNA-binding protein (hnRNP A1) actively participating in SV RNA replication in molecular detail. These studies not only improve our understanding of the replication of SV, but also have the potential for use as the basis for developing antiviral agents that act by inhibiting the virus RNA replication.

\section{Conclusion}

Our previous study and the present report demonstrate that hnRNP A1 is essential for the RNA replication of Sindbis virus. hnRNP A1 binds to the $5^{\prime}$ UTR of SV RNA and facilitates the negative strand RNA synthesis. Moreover hnRNP A1 interacted with the genomic (G) and subgenomic (SG) RNA promoters and is required for the synthesis of G and SG RNA both in infected cells and in vitro. Our study provides the first direct evidence that hnRNP A1 actively participates in viral RNA replication and is required for the synthesis of $G$ and SG RNA.

\section{Acknowledgements}

This work was supported by U.S. Public Health Services Grant Al-70668 from the National Institutes of Health.

\section{Author details}

'Department of Physiology \& Biophysics, Molecular Genetics, Microbiology \& Immunology, UMDNJ-Robert Wood Johnson Medical School, Piscataway NJ 08854, USA. ${ }^{2}$ Department of OB/GYN, UMDNJ-Robert Wood Johnson Medical
School, Piscataway NJ 08854, USA. ${ }^{3}$ Department of Biology \& Molecular

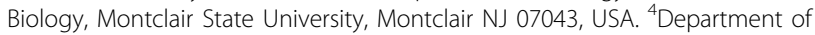
Molecular Genetics, Microbiology \& Immunology, UMDNJ-Robert Wood Johnson Medical School, Piscataway NJ 08854, USA. ${ }^{5}$ The Cancer Institute of New Jersey, New Brunswick NJ 08903, USA

\section{Authors' contributions}

HXG designed the experiments and carried out the in vitro RNA synthesis, virus titration and drafted the manuscript, CWL and SA carried out the Northern and Western blots, SA proof read the manuscript, VS and MLL designed and troubleshoot the experiments, and prepared the manuscript. All authors read and approved the final manuscript.

\section{Competing interests}

The authors declare that they have no competing interests.

Received: 29 April 2010 Accepted: 21 July 2010 Published: 21 July 2010

\section{References}

1. Griffin DE: Alphaviruses. Fields Virology Lippincott Williams \& Wilkinsknipe DM, Howley PM 2007, 1023-1068.

2. Weaver SC, Barrett AD: Transmission cycles, host range, evolution and emergence of arboviral disease. Nat Rev Microbiol 2004, 2(10):789-801.

3. Strauss JH, Strauss EG: The alphaviruses: gene expression, replication, and evolution. Microbiol Rev 1994, 58(3):491-562.

4. Kuhn RJ: Togaviridae. Fields Virology Lippincott Williams \& WilkinsKnipe DM Howley PM 2007, 1001-1022.

5. Shirako Y, Strauss JH: Regulation of Sindbis virus RNA replication: uncleaved P123 and nsP4 function in minus-strand RNA synthesis, whereas cleaved products from P123 are required for efficient plusstrand RNA synthesis. J Virol 1994, 68(3):1874-1885.

6. Ahola T, Kaariainen L: Reaction in alphavirus mRNA capping: formation of a covalent complex of nonstructural protein nsP1 with 7-methyl-GMP. Proc Natl Acad Sci USA 1995, 92(2):507-511.

7. Mi S, Durbin R, Huang HV, Rice CM, Stollar V: Association of the Sindbis virus RNA methyltransferase activity with the nonstructural protein nsP1. Virology 1989, 170(2):385-391.

8. Scheidel LM, Stollar V: Mutations that confer resistance to mycophenolic acid and ribavirin on Sindbis virus map to the nonstructural protein nsP1. Virology 1991, 181(2):490-499.

9. Ahola T, Kujala P, Tuittila M, Blom T, Laakkonen P, Hinkkanen A, Auvinen P: Effects of palmitoylation of replicase protein $\mathrm{nsP1}$ on alphavirus infection. J Virol 2000, 74(15):6725-6733.

10. Wang YF, Sawicki SG, Sawicki DL: Sindbis virus nsP1 functions in negative-strand RNA synthesis. J Virol 1991, 65(2):985-988.

11. Peranen J, Laakkonen $\mathrm{P}$, Hyvonen $\mathrm{M}$, Kaariainen $\mathrm{L}$ : The alphavirus replicase protein nsP1 is membrane-associated and has affinity to endocytic organelles. Virology 1995, 208(2):610-620.

12. Vasiljeva $L$, Merits $A$, Auvinen $P$, Kaariainen $L$ : Identification of a novel function of the alphavirus capping apparatus. RNA 5'-triphosphatase activity of Nsp2. J Biol Chem 2000, 275(23):17281-17287.

13. Gomez dC, Ehsani N, Mikkola ML, Garcia JA, Kaariainen L: RNA helicase activity of Semliki Forest virus replicase protein NSP2. FEBS Lett 1999, 448(1):19-22.

14. Hardy WR, Strauss JH: Processing the nonstructural polyproteins of sindbis virus: nonstructural proteinase is in the C-terminal half of nsP2 and functions both in cis and in trans. J Virol 1989, 63(11):4653-4664.

15. Gorchakov R, Frolova E, Frolov I: Inhibition of transcription and translation in Sindbis virus-infected cells. J Virol 2005, 79(15):9397-9409.

16. Rikkonen M, Peranen J, Kaariainen L: Nuclear and nucleolar targeting signals of Semliki Forest virus nonstructural protein nsP2. Virology 1992, 189(2):462-473.

17. Frolova E, Gorchakov R, Garmashova N, Atasheva S, Vergara LA, Frolov I: Formation of nsP3-specific protein complexes during Sindbis virus replication. J Virol 2006, 80(8):4122-4134.

18. Kamer G, Argos P: Primary structural comparison of RNA-dependent polymerases from plant, animal and bacterial viruses. Nucleic Acids Res 1984, 12(18):7269-7282.

19. Tomar S, Hardy RW, Smith JL, Kuhn RJ: Catalytic core of alphavirus nonstructural protein ns $\mathrm{P} 4$ possesses terminal adenylyltransferase activity. J Virol 2006, 80(20):9962-9969. 
20. Lemm JA, Rumenapf T, Strauss EG, Strauss JH, Rice CM: Polypeptide requirements for assembly of functional Sindbis virus replication complexes: a model for the temporal regulation of minus- and plusstrand RNA synthesis. EMBO J 1994, 13(12):2925-2934.

21. Grdzelishvili VZ, Garcia-Ruiz $H$, Watanabe T, Ahlquist P: Mutual interference between genomic RNA replication and subgenomic mRNA transcription in brome mosaic virus. J Virol 2005, 79(3):1438-1451.

22. Hao L, Sakurai A, Watanabe T, Sorensen E, Nidom CA, Newton MA, Ahlquist $P$, Kawaoka $Y$ : Drosophila RNAi screen identifies host genes important for influenza virus replication. Nature 2008

23. Krishnan MN, Ng A, Sukumaran B, Gilfoy FD, Uchil PD, Sultana H, Brass AL, Adametz R, Tsui M, Qian F, Montgomery RR, Lev S, Mason PW, Koski RA, Elledge SJ, Xavier RJ, Agaisse H, Fikrig E: RNA interference screen for human genes associated with West Nile virus infection. Nature 2008, 455(7210):242-245

24. Sawicki DL, Silverman RH, Williams BR, Sawicki SG: Alphavirus minus-strand synthesis and persistence in mouse embryo fibroblasts derived from mice lacking RNase L and protein kinase R. J Viro/ 2003, 77(3):1801-1811.

25. Sawicki DL, Perri S, Polo JM, Sawicki SG: Role for nsP2 proteins in the cessation of alphavirus minus-strand synthesis by host cells. J Virol 2006, 80(1):360-371

26. Pardigon N, Strauss $\mathrm{JH}$ : Mosquito homolog of the La autoantigen binds to Sindbis virus RNA. J Virol 1996, 70(2):1173-1181.

27. Burnham AJ, Gong L, Hardy RW: Heterogeneous nuclear ribonuclear protein $\mathrm{K}$ interacts with Sindbis virus nonstructural proteins and viral subgenomic mRNA. Virology 2007, 367(1):212-221.

28. Cristea IM, Carroll JW, Rout MP, Rice CM, Chait BT, MacDonald MR: Tracking and elucidating alphavirus-host protein interactions. J Biol Chem 2006, 281(40):30269-30278

29. Atasheva S, Gorchakov R, English R, Frolov I, Frolova E: Development of Sindbis viruses encoding nsP2/GFP chimeric proteins and their application for studying nsP2 functioning. J Virol 2007, 81(10):5046-5057.

30. Spriggs KA, Bushell M, Mitchell SA, Willis AE: Internal ribosome entry segment-mediated translation during apoptosis: the role of IRES-transacting factors. Cell Death Differ 2005, 12(6):585-591.

31. Cammas A, Pileur F, Bonnal S, Lewis SM, Leveque N, Holcik M, Vagner S: Cytoplasmic relocalization of heterogeneous nuclear ribonucleoprotein A1 controls translation initiation of specific mRNAs. Mol Biol Cell 2007, 18(12):5048-5059.

32. Lewis SM, Veyrier A, Hosszu UN, Bonnal S, Vagner S, Holcik M: Subcellular relocalization of a trans-acting factor regulates XIAP IRES-dependent translation. Mol Biol Cell 2007, 18(4):1302-1311

33. Dreyfuss G, Matunis MJ, Pinol-Roma S, Burd CG: hnRNP proteins and the biogenesis of mRNA. Annu Rev Biochem 1993, 62:289-321, 289-321.

34. Weighardt F, Biamonti G, Riva S: The roles of heterogeneous nuclear ribonucleoproteins (hnRNP) in RNA metabolism. Bioessays 1996, 18(9):747-756

35. Carpenter B, McKay M, Dundas SR, Lawrie LC, Telfer C, Murray Gl: Heterogeneous nuclear ribonucleoprotein $\mathrm{K}$ is over expressed, aberrantly localised and is associated with poor prognosis in colorectal cancer. Br J Cancer 2006, 95(7):921-927.

36. Ting NS, Pohorelic B, Yu Y, Lees-Miller SP, Beattie TL: The human telomerase RNA component, hTR, activates the DNA-dependent protein kinase to phosphorylate heterogeneous nuclear ribonucleoprotein A1. Nucleic Acids Res 2009.

37. Shi ST, Huang P, Li HP, Lai MM: Heterogeneous nuclear ribonucleoprotein A1 regulates RNA synthesis of a cytoplasmic virus. EMBO J 2000, 19(17):4701-4711.

38. Kim CS, Seol SK, Song OK, Park JH, Jang SK: An RNA-binding protein, hnRNP A1, and a scaffold protein, septin 6 , facilitate hepatitis $C$ virus replication. J Virol 2007, 81(8):3852-3865.

39. Paranjape $S M$, Harris E: $Y$ box-binding protein-1 binds to the dengue virus 3 '-untranslated region and mediates antiviral effects. J Biol Chem 2007, 282(42):30497-30508

40. Zhao X, Rush M, Schwartz S: Identification of an hnRNP A1-dependent splicing silencer in the human papillomavirus type $16 \mathrm{~L} 1$ coding region that prevents premature expression of the late L1 gene. J Virol 2004, 78(20):10888-10905.

41. Cheunim T, Zhang J, Milligan SG, McPhillips MG, Graham SV: The alternative splicing factor hnRNP A1 is up-regulated during virusinfected epithelial cell differentiation and binds the human papillomavirus type 16 late regulatory element. Virus Res 2008, 131(2):189-198.

42. Wang YF, Chen SC, Wu FY, Wu CW: The interaction between human cytomegalovirus immediate-early gene 2 (IE2) protein and heterogeneous ribonucleoprotein A1. Biochem Biophys Res Commun 1997, 232(3):590-594.

43. Pettit Kneller EL, Connor JH, Lyles DS: hnRNPs Relocalize to the Cytoplasm Following Infection with Vesicular Stomatitis Virus. J Virol 2008.

44. Lin JY, Shih SR, Pan M, Li C, Lue CF, Stollar V, Li ML: hnRNP A1 interacts with the $5^{\prime}$ untranslated regions of enterovirus 71 and Sindbis virus RNA and is required for viral replication. J Virol 2009, 83(12):6106-6114.

45. Lemm JA, Bergqvist A, Read CM, Rice CM: Template-dependent initiation of Sindbis virus RNA replication in vitro. J Virol 1998, 72(8):6546-6553.

46. Strauss EG, De Groot RJ, Levinson R, Strauss JH: Identification of the active site residues in the nsP2 proteinase of Sindbis virus. Virology 1992, 191(2):932-940.

47. Li ML, Stollar V: Identification of the amino acid sequence in Sindbis virus nsP4 that binds to the promoter for the synthesis of the subgenomic RNA. Proc Natl Acad Sci USA 2004, 101(25):9429-9434.

48. Li ML, Stollar V: Distinct sites on the Sindbis virus RNA-dependent RNA polymerase for binding to the promoters for the synthesis of genomic and subgenomic RNA. J Virol 2007, 81(8):4371-4373.

49. Li ML, Kwan TY, Simmonds HA, Stollar V: Synthesis of genomic and subgenomic RNA in mosquito cells infected with two Sindbis virus nsP4 mutants: influence of intracellular nucleoside triphosphate concentrations. J Virol 2008, 82(14):6880-6888

doi:10.1186/1423-0127-17-59

Cite this article as: Gui et al:: hnRNP A1 interacts with the genomic and subgenomic RNA promoters of Sindbis virus and is required for the synthesis of G and SG RNA. Journal of Biomedical Science 2010 17:59.

\section{Submit your next manuscript to BioMed Central and take full advantage of:}

- Convenient online submission

- Thorough peer review

- No space constraints or color figure charges

- Immediate publication on acceptance

- Inclusion in PubMed, CAS, Scopus and Google Scholar

- Research which is freely available for redistribution

Submit your manuscript at www.biomedcentral.com/submit
Biomed Central 